Artículo científico

(Original paper)

\title{
DIVERSIDAD DE COLEOPTERA (INSECTA) EN DOS COMUNIDADES VEGETALES DEL RANCHO TESEACHI, CHIHUAHUA, MÉXICO
}

\section{DIVERSITY OF COLEOPTERA (INSECTA) IN TWO PLANT COMMUNITIES AT THE TESEACHI RANCH, CHIHUAHUA, MEXICO}

\author{
DANIEL OCHOA-GARCÍA ${ }^{1 *}$, JeSÚS A. FERNÁNDEZ ${ }^{1,2}$, VIOLETA SARAÍ JIMÉNEZ-HERNÁNDEZ ${ }^{3}$, ÁNGELA \\ ANDREA CAMARGO-SANABRIA ${ }^{2,4}$, JOHNATTAN HERNÁNDEZ-CUMPLIDO ${ }^{3}$, JOSÉ ROBERTO ESPINOZA \\ PRIETO $^{1}$ km. 1, Chihuahua, Chihuahua, México.<ochoagarciadaniel@gmail.com>; <afernandezf@uach.mx>; <espinozajose1963@gmail.com>
${ }^{2}$ Departamento de Recursos Naturales, Facultad de Zootecnia y Ecología, Universidad Autónoma de Chihuahua. Periférico Francisco R. Almada km 1, Chihuahua, Chihuahua, México. <afernandezf@uach.mx>; <acamargo@uach.mx>
${ }^{3}$ Departamento de Ecología y Recursos Naturales, Universidad Nacional Autónoma de México, Ciudad de México, México. <sarai_aries21@hotmail.com>; <johnattanhdez@ciencias.unam.mx>
${ }^{4}$ CONACYT - Universidad Autónoma de Chihuahua, Facultad de Zootecnia y Ecología, Periférico Francisco R. Almada km 1, Chihuahua, México. <acamargo@uach.mx>
*Autor de correspondencia: <ochoagarciadaniel@gmail.com> \\ ${ }^{1}$ Ingeniería en Ecología, Facultad de Zootecnia y Ecología, Universidad Autónoma de Chihuahua. Periférico Francisco R. Almada
}

Recibido: 21/06/2019; aceptado: 30/09/2019; publicado en línea: 03/10/2019

Editor responsable: Magdalena Cruz Rosales

Ochoa-García, D., Fernández, J. A., Jiménez-Hernández, V. S., Camargo-Sanabria, A. A., Hernández-Cumplido, J., Espinoza, J. R. (2019) Diversidad de Coleoptera (Insecta) en dos comunidades vegetales del rancho Teseachi, Chihuahua, México. Acta Zoológica Mexicana (nueva serie), 35, 1-13. https://doi.org/10.21829/azm.2019.3502213

RESUMEN. Los coleópteros son el orden de insectos más diverso y estudiado del mundo. Sin embargo, el conocimiento de su diversidad en Chihuahua es escaso. En este estudio se comparó la diversidad de coleópteros en dos comunidades vegetales (pastizal de zacate navajita y bosque de encino-pino). Las colectas se realizaron mensualmente en un periodo de seis meses, de junio a noviembre de 2018. Se definieron transectos lineales en ambas comunidades donde se emplearon dos métodos de recolecta. Se determinó la riqueza específica y la abundancia para cada comunidad vegetal. Para evaluar la diversidad de especies de coleópteros para cada tipo de vegetación, se utilizó el método del número de especies efectivas. Para evaluar cómo cambian las abundancias relativas se utilizó una curva de rango-abundancia y se realizó un escalamiento multidimensional no-métrico para evaluar la similitud entre comunidades. Se obtuvo un total de 209 individuos, clasificados en 12 familias, 29 géneros y 42 especies y morfo especies. El mayor número de individuos y de especies se encontró en el bosque de encino-pino. Sin embargo, los índices demostraron que existe una mayor diversidad en los pastizales (16.6 especies efectivas) comparada con los bosques de encino-pino ( 8.5 especies efectivas). La similitud entre comunidades fue baja, lo cual puede estar determinada por la presencia de especies exclusivas asociadas para cada comunidad (20 para el bosque de pino-encino y 14 para el pastizal). Se enlistan 13 especies, siete géneros y una familia como nuevos registros para el estado de Chihuahua.

Palabras clave: Diversidad específica; época de lluvias; norte de México; bosques templados 
Ochoa-García, D., Fernández, J. A., Jiménez-Hernández, V. S., Camargo-Sanabria, A. A., Hernández-Cumplido, J., Espinoza, J. R. (2019) Diversity of Coleoptera (Insecta) in two plant communities at the Teseachi ranch, Chihuahua, Mexico. Acta Zoológica Mexicana (nueva serie), 35, 1-13. https://doi.org/10.21829/azm.2019.3502213

\begin{abstract}
Coleoptera is one of the most diverse and studied order of insects. However, knowledge of its diversity in Chihuahua is scarce. In this study, the diversity of beetles in two plant communities (Navajita grass pasture and oak-pine forest) was compared. The collections were made monthly through a period of six months, from June to November of 2018. Transects were defined in both communities where two methods of collection were used (manual and pitfall traps). We determined the species richness and abundance for each plant community. To assess the diversity of Coleoptera species per vegetation type, a species effective method was performed. To evaluate the change in relative abundances, a range-abundance curve was graphed, and a non-metric multidimensional scaling was performed in order to evaluate the similarity between communities. 209 organisms were obtained, represented by 12 families, 29 genera and 42 morpho species and species. The largest number of individuals and species was found in the Oak-pine forest. However, the index showed that there is greater diversity in grasslands (16.6 effective species) than in the oak-pine forests ( 8.5 effective species). The similarity between communities was low, which may be determined by the presence of associated exclusive species for each type of forest community ( 20 for the pine-oak forest and 14 for the grassland) 13 species, seven genera and one family are listed as new records for the state of Chihuahua.
\end{abstract}

Key words: Species diversity; rain season; northern Mexico; temperate forests

\title{
INTRODUCCIÓN
}

Está ampliamente documentado que México posee una gran diversidad biológica, la cual enfrenta hoy en día graves problemas de conservación (Núñez et al., 2003; Martínez-Meyer et al., 2014). El análisis de la biodiversidad de un área o región contribuye a entender los patrones y procesos, así como el papel que tiene cada especie en los ecosistemas (Thomas, 2013; Vellend, 2017). Su estudio implica registrar, caracterizar, comprender, cuantificar, cualificar y preservar organismos de vida silvestre (Martínez-Meyer et al., 2014). Uno de los grupos más estudiado y numeroso del mundo es el de los insectos. Según McGavin (2002), el $55.3 \%$ de los organismos que habitan en el planeta son insectos, con 29 órdenes, 949 familias y más de un millón de especies descritas. Los coleópteros son el grupo más diverso del Orden Insecta (Zhang et al., 2018), cuenta con 392,415 especies descritas, incluyendo 2,928 especies extintas (Zhang, 2013). Los coleópteros han sido sumamente exitosos colonizando diferentes tipos de hábitat, desde áreas terrestres hasta cuerpos de agua dulce (Triplehorn \& Johnson, 2005), lo que hace de ellos un grupo de importancia biológica y ecológica (McGavin, 2002), y fundamental para evaluar la calidad de los ecosistemas y su conservación (Ribera \& Foster, 1997; Fontúrbel, 2002; Zhang, 2013; Guzmán-Mendoza et al., 2016).

La vegetación y sus características pueden influir en la diversidad de los coleópteros (Escobar, 2000; Sanabria et al., 2008; Jiménez-Sánchez et al., 2013; Sánchez-Reyes et al., 2015; Sánchez-Hernández et al., 2018), por eso la importancia de muestrear varios tipos de vegetación ya que con monitoreos extensos podremos obtener una visión amplia de los organismos que se encuentran en nuestros ecosistemas y así contribuir a su conservación.

Para Chihuahua, el conocimiento de la fauna de coleópteros es escaso. Los primeros registros para el estado fueron realizados por Furth y Jolivet (2009), quienes reportaron 68 especies, 24 géneros y una familia (Chrysomelidae). Dos años después, García et al., (2011) listaron 15 especies, diez géneros y dos familias (Melolonthidae y Scarabaeidae). El Instituto de Biología (IBUNAM) en su Portal de Datos Abiertos UNAM (2014) muestra datos de especímenes recolectados que representan 17 especies, diez géneros y seis 
familias más (Cantharidae, Geotrupidae, Lamparydae, Lycidae, Meloidae, Telegeusidae). Finalmente, en las bases de datos de la Comisión Nacional para el Conocimiento y Uso de la Biodiversidad (CONABIO, 2014) se reportan cinco especies más: el pinacate (Eleodes obscurus LeConte, 1851), el botijón (Meloe sp.), la mariquita (Hippodamia convergens Guérin-Méneville, 1842), el escarabajo de cuadros cleritos (Necrobia spp.) y la doradilla (Diabrotica undecimpunctata Mannerheim, 1843). Actualmente, la fauna de coleópteros reportada en el estado de Chihuahua está integrada por 105 especies, pertenecientes a 51 géneros y 14 familias.

Para El Rancho Teseachi ubicado en la parte centro-sur del estado, entre los municipios de Namiquipa, Bachíniva y Guerrero, este trabajo constituye un aporte a la actualización del conocimiento de los Insecta: Coleoptera. Los objetivos de este trabajo fueron: 1) generar un listado de especies de coleópteros de El Rancho Teseachi y 2) comparar la diversidad de coleópteros en dos comunidades vegetales (pastizal de zacate navajita y bosque de encino-pino).

\section{MATERIALES Y MÉTODOS}

Área de estudio. El Centro de Investigación y Transferencia de Tecnología Teseachi "CEITT Teseachic" se localiza en el estado de Chihuahua entre los municipios de Namiquipa, Bachíniva y Guerrero (Fig. 1). Teseachi está ubicado geográficamente entre las coordenadas $28^{\circ} 47^{\prime} 42.24^{\prime \prime} \mathrm{N}$; $107^{\circ} 26^{\prime} 06.79$ ' $\mathrm{O}$, con una altitud media de $2,083 \mathrm{~m}$; tiene un clima seco-árido (Bs1 kw), con una temperatura media anual de $13^{\circ} \mathrm{C}$, la cual puede variar de $-5.9^{\circ} \mathrm{C}$ a $31.6^{\circ} \mathrm{C}$; la precipitación pluvial promedio anual es de $580 \mathrm{~mm}$ (Trevizo, 2016) y la mayor parte de esta se precipita en los meses de junio-septiembre. Abarca una superficie total de 12,300 ha de las cuales alrededor de 300 ha tienen potencial agrícola y las restantes se dividen en 15 secciones (Alamillo, Área agrícola, Bajío Ancho, Casco, El Estudiante, Fistulados, La Selva, Lajas, Las Coloradas, Palmitas, Potrero Nuevo, Rancho Viejo, Remuda, Savory y Varitas; Cuadro 1), con la finalidad de facilitar el manejo del rancho. La vegetación se caracteriza por la presencia de pastizales de zacate navajita (Bouteloua gracilis (Kunth) Lag. Ex Steud), bosque de encino (Quercus sp.) y bosque de encinopino (Quercus-Pinus) (UACH, 2011).

Cuadro 1. Localización (coordenadas geográficas y altitud) de los sitios muestreados en dos comunidades (pastizal natural y encino-pino) de El Rancho Teseachi, Chihuahua, México.

\begin{tabular}{|c|c|c|c|}
\hline LOCALIDADES & LATITUD & LONGITUD & ALTITUD \\
\hline \multicolumn{4}{|l|}{ Bosque de encino-pino } \\
\hline Lajas & $28^{\circ} 46^{\prime} 24.39^{\prime \prime} \mathrm{N}$ & $107^{\circ} 27^{\prime} 33.36^{\prime \prime} \mathrm{O}$ & $2,198 \mathrm{~m}$ \\
\hline Bajío Ancho & $28^{\circ} 48^{\prime} 26.41^{\prime \prime} \mathrm{N}$ & $107^{\circ} 26^{\prime} 37.85^{\prime \prime} \mathrm{O}$ & $2,164 \mathrm{~m}$ \\
\hline La Selva & $28^{\circ} 47^{\prime} 23.15^{\prime \prime} \mathrm{N}$ & $107^{\circ} 26^{\prime} 59.81^{\prime \prime} \mathrm{O}$ & $2,209 \mathrm{~m}$ \\
\hline El Casco & $28^{\circ} 53^{\prime} 24.48^{\prime \prime} \mathrm{N}$ & $107^{\circ} 27^{\prime} 14.98^{\prime \prime} \mathrm{O}$ & $1,990 \mathrm{~m}$ \\
\hline Alamillo & $28^{\circ} 47^{\prime} 24.42^{\prime \prime} \mathrm{N}$ & $107^{\circ} 26^{\prime} 55.29^{\prime \prime} \mathrm{O}$ & $2,208 \mathrm{~m}$ \\
\hline \multicolumn{4}{|l|}{ Pastizal de zacate navajita } \\
\hline Lajas & $28^{\circ} 46^{\prime} 51.19^{\prime \prime} \mathrm{N}$ & $107^{\circ} 27^{\prime} 49.56^{\prime \prime} \mathrm{O}$ & $2,164 \mathrm{~m}$ \\
\hline Bajío Ancho & $28^{\circ} 48^{\prime} 59^{\prime} 76^{\prime \prime} \mathrm{N}$ & $107^{\circ} 26^{\prime} 45.60^{\prime \prime} \mathrm{O}$ & $2,080 \mathrm{~m}$ \\
\hline La Selva & $28^{\circ} 47^{\prime} 56.30^{\prime \prime} \mathrm{N}$ & $107^{\circ} 27^{\prime} 39.62^{\prime \prime} \mathrm{O}$ & $2,089 \mathrm{~m}$ \\
\hline El Casco & $28^{\circ} 53^{\prime} 00.32^{\prime \prime} \mathrm{N}$ & $107^{\circ} 26^{\prime} 56.87^{\prime \prime} \mathrm{O}$ & $1,952 \mathrm{~m}$ \\
\hline Alamillo & $28^{\circ} 47^{\prime} 14.64^{\prime \prime} \mathrm{N}$ & $107^{\circ} 26^{\prime} 24.52^{\prime \prime} \mathrm{O}$ & $2,140 \mathrm{~m}$ \\
\hline
\end{tabular}

Para realizar el muestreo se seleccionaron cinco secciones: Alamillo, Bajío Ancho, El Casco, La Selva y Lajas, con una separación aproximada de 0.5-1.0 km. En cada sección se encontraba un sitio de pastizal de zacate navajita y otro de bosque de encino-pino (Fig.1; Cuadro 1), estas secciones en conjunto representan el 59\% de la superficie del Rancho Teseachi (CONABIO, 2018). Cabe destacar que debido a 
que una parte del muestreo se realizó en temporada de lluvias, se coincidió con la época de floración de varias especies de plantas compuestas, especialmente en los pastizales, lo que favoreció la presencia de insectos polinizadores.

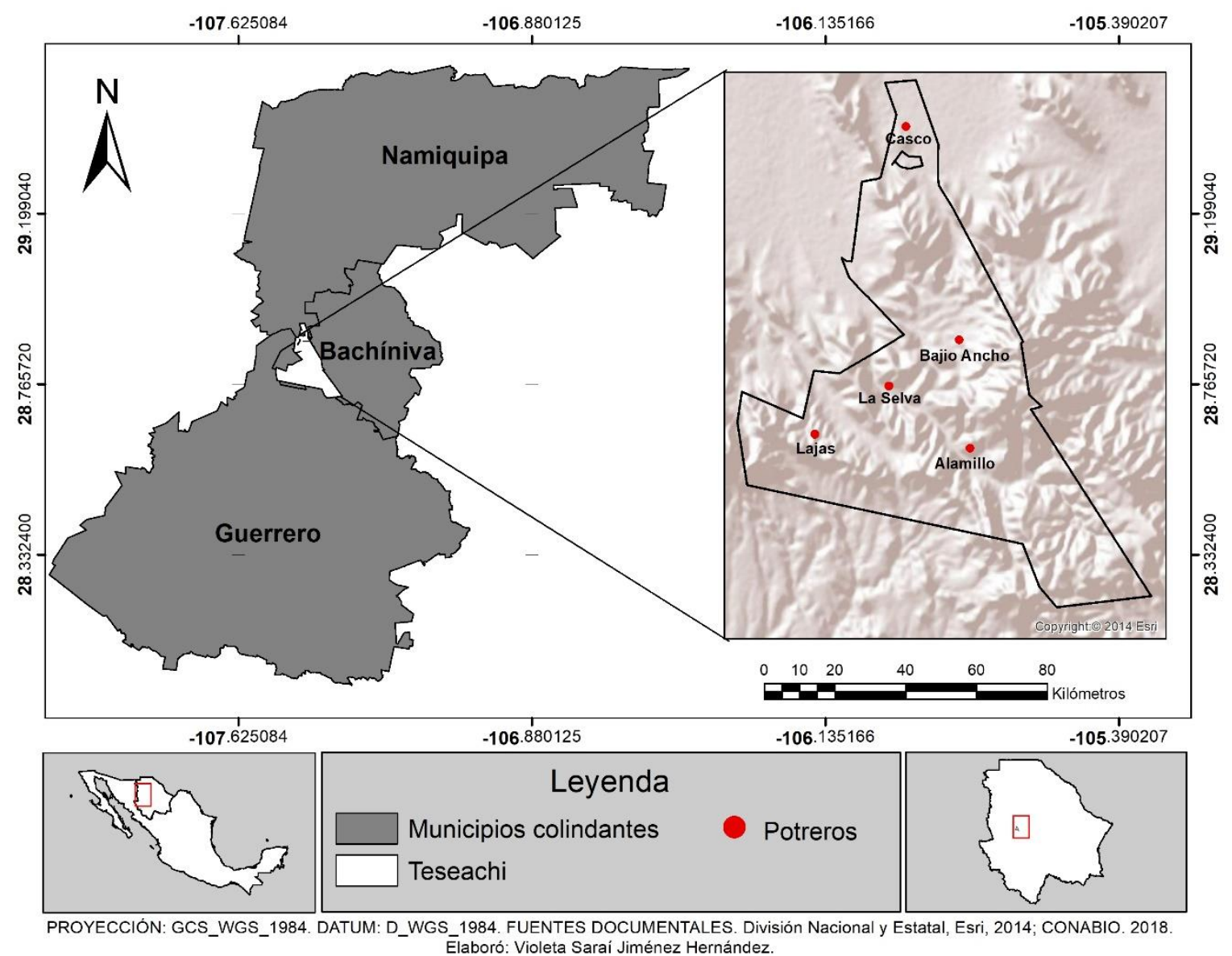

Figura 1. Ubicación geográfica del área de estudio en el estado de Chihuahua, México. Los puntos señalan los cinco potreros muestreados.

Muestreo. La recolección de los individuos se realizó a través de seis muestreos mensuales durante cuatro meses en la temporada de lluvias (junio-septiembre) y dos en la de transición (octubre y noviembre) de 2018. Se emplearon dos métodos de captura de acuerdo con Márquez-Luna (2005): 1) transectos de colecta manual, 2) trampas de caída o "pitfall". Las trampas de caída consistieron en un recipiente de plástico de un litro de capacidad enterradas a ras del suelo, con $200 \mathrm{ml}$ de alcohol al $70 \%$ y estiércol de perro como atrayente (Cárdenas \& Hidalgo, 2006). Las trampas se colocaron sobre un transecto lineal de $300 \mathrm{~m}$ de longitud distanciadas $50 \mathrm{~m}$ una de otra. Las trampas estuvieron activas $24 \mathrm{~h}$ y se revisaron dos veces durante ese periodo de tiempo. Se emplearon dos transectos de colecta manual de $50 \mathrm{~m}$ de longitud, uno para el pastizal y otro para el bosque de encino-pino dentro de cada sección. En estos transectos se realizó la búsqueda directa de los organismos en las dos comunidades vegetales de cada sección. Una vez localizados los organismos se capturaron con pinzas entomológicas y se depositaron en frascos con alcohol etílico al $70 \%$. Para evitar una subestimación de los resultados, los dos transectos de búsqueda manual establecidos en cada sección estuvieron separados $200 \mathrm{~m}$ y se hicieron de manera paralela al sitio establecido para las trampas de caída. A cada transecto se le dedicaron cuatro horas, en un horario establecido entre las 8:00 y 15:00 horas durante el tiempo en que las trampas de caída estuvieron activas. 
La identificación taxonómica se realizó bajo un microscopio estereoscópico VE-153G, utilizando las claves de Borror y Delongs (Triplehorn \& Johnson, 2005), American Beetles Volumen I y II (Arnett \& Thomas, 2000; Arnett, et al., 2002) y con la ayuda de personal del Instituto de Biología, UNAM. Los ejemplares fueron depositados en la Facultad de Zootecnia y Ecología de la UACH en el Laboratorio de Recursos Naturales.

Análisis de datos. La riqueza de especies se definió como el número de especies encontradas en cada comunidad vegetal. La diversidad de especies para cada comunidad vegetal se evaluó a partir del uso del número de especies efectivas propuesto por Jost (2006). Para realizar este análisis se utilizó la diversidad de orden $1\left({ }^{l} D\right)$, que es el exponencial de la entropía del índice de Shannon (diversidad verdadera). El orden $\left({ }^{1} D\right)$ permite incluir todas las especies proporcionalmente a su abundancia en la comunidad (Moreno et al., 2011; Gotelli \& Chao, 2013). La abundancia relativa de las especies se estimó como el número de individuos de cada especie presente en cada sitio entre el total de individuos de todas las especies, la cual fue contabilizada por sección y comunidad vegetal. Se usaron curvas de rango-abundancia para comparar la distribución de las abundancias de las especies entre comunidades (Moreno et al., 2011). Para comparar la composición de especies entre comunidades se utilizaron las abundancias relativas y realizó un escalamiento multidimensional no-métrico (NMDS, McCune \& Grace, 2002) y un análisis de similitud (ANOSIM). El NMDS mide a través de la función de estrés, escalada entre 0 y 1 , qué tan lejanas son las distancias en el espacio multidimensional de las originales en una matriz de asociación (Borcard et al., 2011).

\section{RESULTADOS Y DISCUSIÓN}

\section{Riqueza de especies}

Se registraron 209 individuos, de los cuales 197 se utilizaron para los análisis, estos están agrupados en 12 familias, 29 géneros y 42 especies y morfoespecies. La familia Scarabaeidae tuvo la mayor riqueza (diez especies) y abundancia (104 individuos), en cambio, Coccinellidae, Curculionidae, Lampyridae, Melyridae y Tenebrionidae fueron las familias con la menor riqueza y abundancia (Cuadro 2). Estos contrastes podrían deberse tanto a las características de los sitios como al tipo de muestreo y los cebos utilizados, ya que para familias como Curculionidae y Lampyridae se necesita establecer trampas o métodos de colecta específicos para lograr su recolección (Sánchez-Soto, 2011; Zaragoza-Caballero, 2015; Pérez-De la Cruz et al., 2016). La familia Erotylidae, los géneros Acmaeodera, Calligrapha, Gibbifer, Harpalus, Odontota, Panagaeus y Pasimachus y las especies Acmaeodera mudgei Westcott, 2002, Calligrapha serpentina Rogers, 1856, Cyclocephala barrerai Martinez, 1969, Gibbifer californicus LaCordaire, 1842, Harpalus rufipes Degeer, 1774, Odontota signaticollis Baly, 1886, Panagaeus sallei Chaudoir, 1862, Pasimachus viridans LeConte, 1858, Chauliognathus misellus Horn, 1885, Chauliognathus profundus LeConte, 1858 у Photinus anisodrilus Zaragoza, 2007 representan nuevos registros para el estado de Chihuahua.

La especie Canthon humectus Say, 1832 fue la más abundante con el $46 \%$ del total de los individuos capturados, mientras que Acmaeodera sp. 4, Calligrapha serpentina, Chlaenius sp., Chrysina sp., Euphoria monticola Bates, 1889, O. signaticollis, Onthophagus sp., Phanaeus sp., y Phyllophaga sp., representaron el $0.5 \%$ del total de la muestra (Cuadro 2). Halffter et al. (2015) afirman que C. humectus se encuentra en un proceso activo de expansión geográfica ya que es una especie común que se presenta en zonas en las que no se tienen registros previos. Esta alta abundancia de $C$. humectus coincide con lo encontrado en otros estudios en hábitats similares al presente estudio (Briseño, 2012; Zamora-Vuelvas et al., 2014; Halffter et al., 2015; Carrillo-Ruiz et al., 2017). 
Cuadro 2. Listado de taxa de coleópteros y número de individuos colectados en El Rancho Teseachi, Chihuahua, México, de junio a noviembre de 2018. "Nuevos registros para el estado.

\begin{tabular}{|c|c|c|c|}
\hline Especies & Bosque de encino-pino & $\begin{array}{c}\text { Pastizal de zacate } \\
\text { navajita }\end{array}$ & Total \\
\hline \multicolumn{4}{|l|}{ Carabidae } \\
\hline Pasimachus viridans Leconte, $1858^{*}$ & 2 & & 2 \\
\hline Harpalus rufipes Degeer, $1774^{*}$ & 2 & 2 & 4 \\
\hline Panagaeus sallei Chaudoir, $1862^{*}$ & 2 & & 2 \\
\hline Brachinus sp. & 7 & & 7 \\
\hline Chlaenius sp. & & 1 & 1 \\
\hline Polpochila sp. & & 3 & 3 \\
\hline Platynus sp. & 5 & & 5 \\
\hline Platynus sp. 2 & 2 & & 2 \\
\hline \multicolumn{4}{|l|}{ Scarabaeidae } \\
\hline Cyclocephala barrerai Martinez, $1969^{*}$ & & 1 & 2 \\
\hline Phanaeus quadridens Say, 1835 & 3 & & 3 \\
\hline Phanaeus sp. & & 1 & 1 \\
\hline Canthon humectus Say, 1832 & 91 & & 91 \\
\hline Xyloryctes sp. & 2 & & 2 \\
\hline Chrysina sp. & 1 & & 1 \\
\hline Onthophagus sp. & 1 & & 1 \\
\hline Euphoria montícola Bates, 1889 & 1 & & 1 \\
\hline Phillophaga sp. & & 1 & 1 \\
\hline Dichotomius sp. & & 2 & 2 \\
\hline \multicolumn{4}{|l|}{ Chrysomelidae } \\
\hline Odontota signaticollis Baly, $1886^{*}$ & 1 & & 1 \\
\hline Calligrapha serpentina Rogers, $1856^{*}$ & & 1 & 1 \\
\hline Diabrotica undecimpunctata & & & 2 \\
\hline Mannerheim, 1843 & & 2 & 2 \\
\hline Leptinotarsa rubiginosa Rogers, 1856 & 2 & & 2 \\
\hline Leptinotarsa sp.1 & 3 & 1 & 4 \\
\hline Leptinotarsa sp. 2 & 2 & 1 & 3 \\
\hline \multicolumn{4}{|l|}{ Lampyridae } \\
\hline Photinus anisodrilus Zaragoza, $2007^{*}$ & 2 & & 2 \\
\hline \multicolumn{4}{|l|}{ Buprestidae } \\
\hline Acmaeodera mudgei Westcott, 2002* & 1 & 2 & 3 \\
\hline Acmaeodera sp. 1 & & 5 & 5 \\
\hline Acmaeodera sp. 2 & & 2 & 2 \\
\hline Acmaeodera sp. 3 & & 5 & 5 \\
\hline Acmaeodera sp. 4 & & 1 & 1 \\
\hline \multicolumn{4}{|l|}{ Erotylidae $^{*}$} \\
\hline Gibbifer californicus LaCordaire, $1842^{*}$ & 3 & & 3 \\
\hline \multicolumn{4}{|l|}{ Cantharidae } \\
\hline Chauliognathus misellus Horn, $1885^{*}$ & 1 & & 1 \\
\hline Chauliognathus profundus LeConte, $1858^{*}$ & & 1 & 1 \\
\hline Chauliognathus sp. & 1 & 9 & 10 \\
\hline Chauliognathus sp. 2 & 5 & & 5 \\
\hline \multicolumn{4}{|l|}{ Meloidae } \\
\hline Nemognatha sp. & 1 & & 1 \\
\hline Nemognatha sp. 2 & 2 & & 2 \\
\hline Lytta sp. & & 2 & 2 \\
\hline \multicolumn{4}{|l|}{ Melyridae } \\
\hline Collops sp. & 3 & & 3 \\
\hline \multicolumn{4}{|l|}{ Tenebrionidae } \\
\hline Eleodes armatus LeConte, 1858 & 3 & 1 & 4 \\
\hline
\end{tabular}




\begin{tabular}{lccc}
\hline Especies & Bosque de encino-pino & $\begin{array}{c}\text { Pastizal de zacate } \\
\text { navajita }\end{array}$ & Total \\
\hline $\begin{array}{l}\text { Coccinellidae } \\
\text { Hippodamia convegens Guérin-Méneville, } 1842\end{array}$ & 2 & 1 & 3 \\
\hline $\begin{array}{l}\text { Curculionidae } \\
\text { Sp. 2 }\end{array}$ & & 1 & \\
\hline TOTAL & 151 & 46 & 197 \\
\hline
\end{tabular}

\section{Composición de especies}

Las comunidades de coleópteros en los dos tipos de vegetación difieren en el número de especies y en la forma en la que se distribuye la abundancia de cada una de ellas (Cuadro 2). Las especies con la mayor abundancia relativa en el bosque de encino-pino fueron: $C$. humectus $(0.46)$, Brachinus sp. (0.03) y Platynus sp. (0.02), mientras que en el pastizal de zacate navajita, las especies con mayor abundancia relativa fueron: Chauliognathus sp. (0.04), Acmaeodera sp. (0.02) y Acmaeodera sp3 (0.02) (Fig. 2).

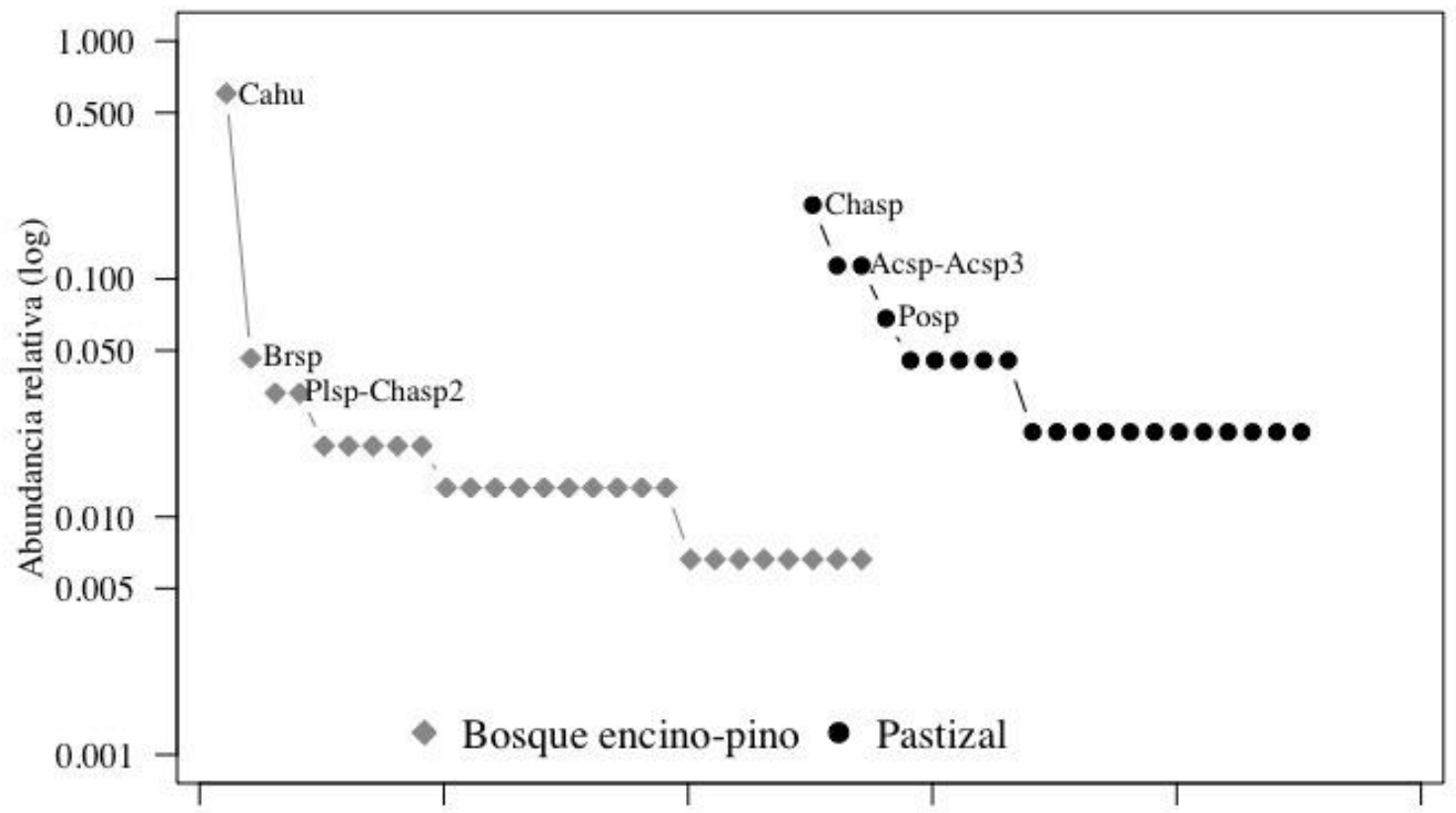

Orden de las especies

Figura 2. Curva de rango-abundancia de dos comunidades de escarabajos muestreados en dos hábitats en El Rancho Teseachi, Chihuahua, México. Cahu=C. humectus, Brsp= Brachinus sp, Plat= Platynus $\mathrm{sp}$, Chau2= Chauliognathus; Chasp $=$ Chauliognathus $\mathrm{sp}$, Acsp= Acmaeodera $\mathrm{sp}$, Acsp3 $=$ Acmaeodera $\mathrm{sp} 3$, Posp= Polpochila $\mathrm{sp}$.

La mayor riqueza de especies y abundancia (Cuadro 2,3) se registraron en el bosque de encinopino (151 individuos y 29 especies), lo cual representa el 77\% del total de los ejemplares y el 69\% del total de las especies. Este tipo de bosque también contiene el $92 \%$ de las familias reportadas en el trabajo. Por otra parte, en el pastizal de zacate navajita hubo menor riqueza (22 especies, $31 \%$ del total), menor abundancia (46 individuos, $23 \%$ del total) y menor número de familias $(n=9)$. Sin embargo, la diversidad verdadera encontrada en el bosque de encino-pino fue de 8.5 especies efectivas, mientras que en el pastizal aumentó a 16.6 especies efectivas (Cuadro 3). Es decir, que en el pastizal de zacate navajita se registró casi 
el doble de la diversidad de coleópteros comparada con el que se registró en el bosque de encino-pino (magnitud de cambio es de 7.6 especies). Cabe resaltar la influencia que tiene la presencia de una especie altamente dominante en el bosque de encino-pino. Si $C$. humectus fuera eliminado del análisis, la diversidad verdadera del bosque de encino-pino sería de 27.3 especies efectivas $\left(H^{\prime}=3.31\right)$ y la magnitud de cambio pasaría de 7.6 especies a 11.2 especies, dejando al bosque de encino-pino como la comunidad más diversa.

La riqueza de especies y la abundancia de Coleoptera estuvieron repartidas de forma homogénea en los pastizales, lo que se refleja en una mayor equidad (Cuadro 3). Coincidiendo con lo encontrado por MejíaNarváez (2018), quien concluye que la mayor diversidad de coleópteros se encuentra en sistemas silvopastoriles. Sin embargo, en el bosque de encino-pino se cuenta con gran abundancia, debido a la presencia de la especie dominante C. humectus (Scarabaeidae), que representa el $60 \%$ de los individuos capturados y se encuentra asociada exclusivamente a este tipo de vegetación. Su ausencia en el pastizal podría deberse a los componentes del ambiente local (temperatura, precipitación, estacionalidad y características del suelo), así como a la disponibilidad de alimento y zonas óptimas de crecimiento y apareamiento (Trevilla-Rebollar et al., 2010). Es oportuno mencionar que esta especie tiene hábitos coprófagos (Halffter et al., 2015) y puede estar siendo afectada negativamente por la ivermectina $\left(\mathrm{C}_{48} \mathrm{H}_{74} \mathrm{O}_{14}\right)$, un desparasitante usado frecuentemente en el ganado vacuno en la región (Thorsten \& Moon, 2019). La utilización de este químico tiene efectos negativos en la abundancia y diversidad de múltiples especies; por ejemplo, Iglesias et al. (2005) encontraron reducciones en la abundancia y diversidad de artrópodos coprófagos como resultado del efecto negativo que la ivermectina tuvo sobre la colonización natural de la materia fecal y la duración del proceso de degradación. Así mismo, Baena-Díaz et al. (2018) determinaron que el desparasitante ivermectina afectó a los individuos del escarabajo del estiércol (Euoniticellus intermedius) y su descendencia. Por lo tanto, es posible que también en nuestro sistema la ivermectina esté alterando la abundancia y diversidad de C. humectus y de otras especies.

Por otro lado, existen estudios que muestran que los bosques de encino-pino albergan la mayor riqueza de Scarabaeidae respecto a la selva mediana subperenifolia y el pastizal en el estado de Veracruz (Gómez-Beda, 2013), lo cual coincide con nuestros resultados. Esto se debe probablemente a que las condiciones de temperatura y humedad que caracterizan estos ecosistemas los hacen hábitats deseables para diversos organismos (Arizaga, 2009), ofreciendo así, refugio y alimento a los coleópteros.

El género Acmaeodera (Buprestidae) fue el más abundante en los pastizales. Su alta abundancia en la temporada muestreada puede estar relacionada con la presencia de plantas en floración durante esta época del año. Se sabe que las especies de este taxón se alimentan de polen y de los pétalos de las flores, principalmente (Romero-Nápoles \& Westcott, 2017).

Cuadro 3. Datos de diversidad de las diferentes comunidades forestales de El Rancho Teseachi, Chihuahua, México.

\begin{tabular}{lcc}
\hline & Bosque encino-pino & Pastizal de zacate navajita \\
\hline Riqueza de especies & 29 & 22 \\
Abundancia & 151 & 46 \\
Shannon-Wiener $\left(H^{\prime}\right)$ & 2.14 & 2.78 \\
Diversidad verdadera & 8,5 & 16.6 \\
Equidad de Pielou $\left(J^{\prime}\right)$ & 0.60 & 0.90 \\
\hline
\end{tabular}

\section{Similitud entre las comunidades}

Se encontró una baja similitud entre las dos comunidades. La agrupación de los sitios coincidió con el tipo de comunidad presentándose dos grandes grupos (bosque encino-pino y pastizal) (Fig. 3). Los resultados del ANOSIM mostraron diferencias significativas entre las dos comunidades (ANOSIM R=0.39, $p<0.03$ ). Las diferencias entre las comunidades vegetales parecen explicarse por la presencia de especies exclusivas, 
por ejemplo, en el bosque de encino-pino: C. humectus, G. californicus, P. viridans y $C$. misellus; en el pastizal, Acmaeodera sp., Lytta sp., C. barrerai, Phillophaga sp. y C. serpentine. En cambio, siete especies (Acmaeodera mudgei, Chauliognathus sp., Eleodes armatus, Harpalus rufipes, Hippodamia convegens, Leptinotarsa sp.1 y Leptinotarsa sp.2) se compartieron en ambas comunidades (Fig. 3). Según Gómez et al. (2015), los sistemas sin estrato arbóreo (pastizales, entre otros) tienden a mantener una riqueza y abundancia de especies alta, pero la tasa de recambio de especies con otros hábitats tiende a ser baja, es decir poseen gran número de especies exclusivas. Por otro lado, Mejía-Narváez (2018) menciona que la similitud de especies entre bosques y pastizales es muy baja (40\% de similitud), estos dos estudios coinciden con lo expuesto en este trabajo, ya que entre las dos comunidades vegetales que se muestrearon se comparten muy pocas especies.

Las familias presentes en ambas comunidades fueron: Carabidae, Chrysomelidae, Cantharidae y Tenebrionidae, en cambio, Lampyridae, Erotylidae y Tenebrionidae solo se encontraron asociadas al bosque de encino-pino y Curculionidae al pastizal.

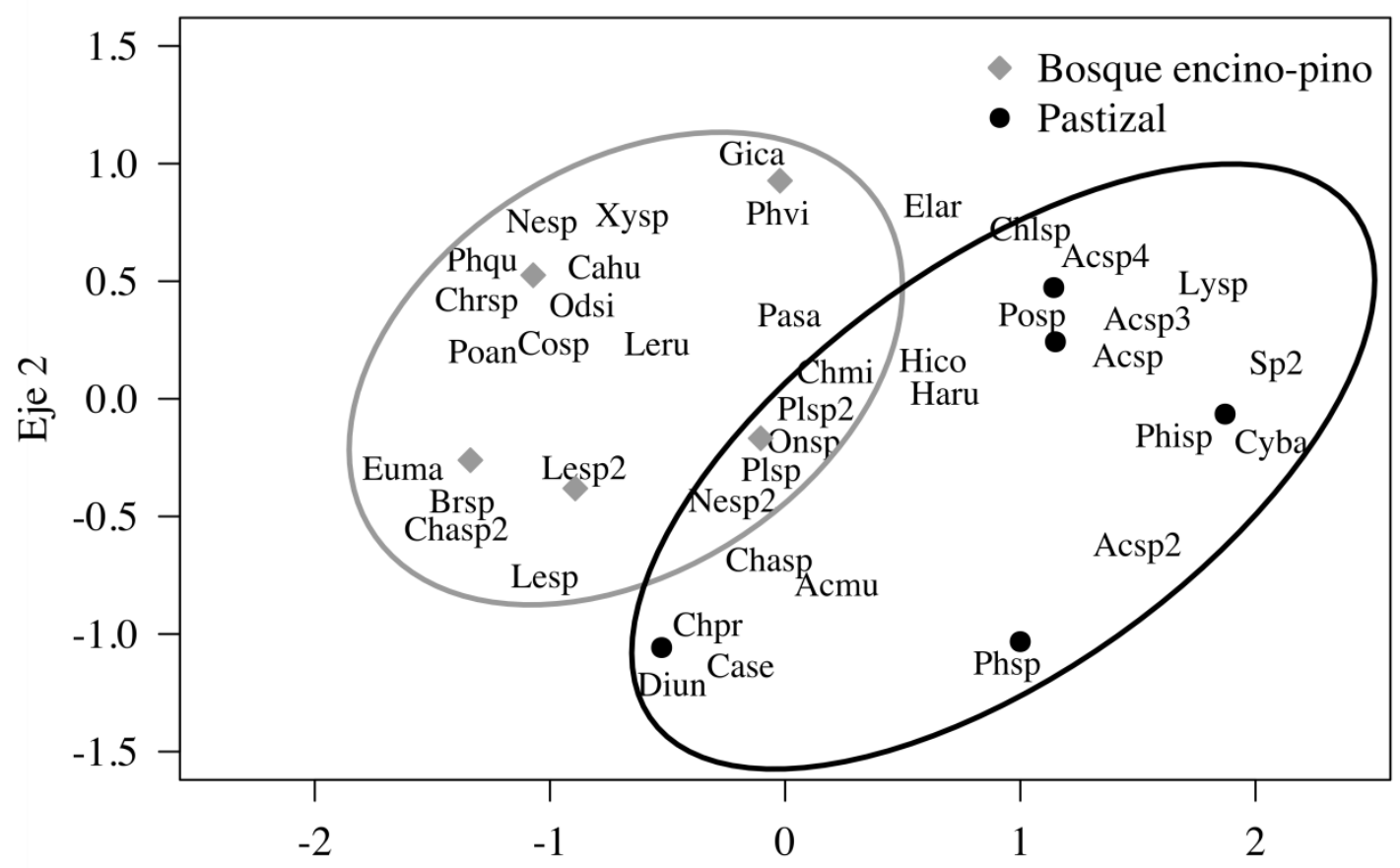

Eje 1

Figura 3. Escalamiento multidimensional no métrico (NMDS) utilizando el Índice de Jaccard. C. humectus= Cahu; Brachinus sp. $=$ Brsp; Platynus sp. $=$ Plsp; Chauliognathus $\mathrm{sp} 2=$ Chasp2; $P$. quadridens $=\mathrm{Phqu} ;$ Leptinotarsa $\mathrm{sp} .=$ Lesp; G. californicus $=$ Gica; Collops sp.= Cosp; E. armatus $=$ Elar; $P$. viridans $=$ Phvi; $H$. rufipes $=$ Haru; $P$. sallei= Pasa; Platynus $\mathrm{sp} 2=\mathrm{Plsp} 2 ;$ Xyloryctes $\mathrm{sp} .=$ Xysp; L. rubiginosa $=$ Leru; Leptinotarsa $\mathrm{sp} 2=\mathrm{Lesp} 2 ;$ $P$. anisodrilus= Poan; Nemognatha $\mathrm{sp} 2=$ Nesp $;$ H. convegens $=$ Hico Chrysina $\mathrm{sp} .=$ Chrsp; Onthophagus $\mathrm{sp} .=$ Onsp E. montícola= Euma; O. signaticollis $=$ Odsi; A. mudge $=$ Acmu; C. misellus $=$ Chmi; Chauliognathus $\mathrm{sp} .=$ Chasp; Nemognatha $\mathrm{sp} .=$

Nesp; Acmaeodera sp.= Acsp; Acmaeodera sp3= Acsp3; Polpochila sp.= Posp; D. undecimpunctata $=$ Diun; Acmaeodera $\mathrm{sp} 2=$ Acsp2; Lytta sp.= Lysp; Chlaenius sp.= Chlsp; C. barrerai $=$ Cyba; Phanaeus $\mathrm{sp} .=\mathrm{Phsp}$; Phillophaga $\mathrm{sp} .=$ Phisp; $C$. serpentina $=$ Case; Acmaeodera $\mathrm{sp} 4=\mathrm{Acsp} 4 ;$. profundus $=\mathrm{Chpr} ; \mathrm{Sp} 2=\mathrm{Sp} 2$. 


\section{CONCLUSIONES}

Se reportan diez especies, siete géneros y una familia como nuevos registros para el estado de Chihuahua, incrementando el estado del conocimiento de los coleópteros a 115 especies, 58 géneros y 15 familias. La mayor diversidad de coleópteros se encontró en los pastizales de zacate navajita.

La familia Scarabaeidae tuvo la mayor riqueza de especies y estuvo presente en las dos comunidades forestales. Canthon hummectus fue la especie más común en el bosque de encino-pino, por lo que puede ser una especie que indique la calidad y salud del ecosistema.

El bosque de encino-pino presentó el mayor número de especies exclusivas (20) en comparación con el pastizal (15). Siete especies se compartieron en ambas comunidades.

Se recomienda realizar análisis en los cuales se evalúe el porcentaje de ivermectina en el cuerpo de los escarabajos y los efectos sobre los individuos y su abundancia.

Agradecimientos. Al Dr. S. Zaragoza Caballero por su ayuda en la identificación taxonómica del grupo. A R. Solís, F. Álvarez y C. Lerma por su apoyo en las colectas. A M. Cruz Caballero y C. Ruiz Rodríguez por el tiempo invertido en este proyecto.

\section{LITERATURA CITADA}

Arizaga, S. (2009) Manual de la biodiversidad de encinos michoacanos. Instituto Nacional de Ecología, $146 \mathrm{pp}$.

Arnett, R. H., Thomas, M. C. (2000) American Beetles, Volume I: Archostemata, Myxophaga, Adephaga, Polyphaga: Staphyliniformia. CRC Press, 464 pp. https://doi.org/10.1201/9781482274325

Arnett, R. H., Frank, J. H., Thomas, M. C., Skelley, P. E. (2002) American Beetles, Volumen II: Polyphaga: Scarabaeoidea through Curculionoidea. CRC press, $861 \mathrm{pp}$.

Baena-Díaz, F., Martínez-M. I., Gil-Pérez, Y., González-Tokman, D. (2018) Trans-generational effects of ivermectin exposure in dung beetles. Chemosphere, 202, 637-643. https://doi.org/10.1016/j.chemosphere.2018.03.109

Borcard, D., Gillet, F., Legendre, P. (2011) Unconstrained ordination. En: Numerical ecology with R. Springer, New York, NY. https://doi.org/10.1007/978-1-4419-7976-6_5

Briseño, V. L. (2012) Análisis de la comunidad de escarabajos coprófagos de Villa de Cos, Zacatecas, México. (Tesis de Doctorado), Universidad Nacional Autónoma de México.

Cárdenas, A. M., Hidalgo, J. M. (2006) Anthicidae del parque nacional de Doñana (España). Boletín Sociedad Entomológica Aragonesa, 1, 173-176.

Carrillo-Ruiz, H., Guerra-González, I., Sánchez-Carrillo, M., Morón, M. A., Rivas-Arancibia, S. P. (2017) Fauna de Scarabaeoidea (Insecta: Coleoptera) de Calmeca, Tepexco, Puebla, México. Acta Zoológica Mexicana (nueva serie), 33, 251-265. https://doi.org/10.21829/azm.2017.3321065

CONABIO (2014) La biodiversidad en Chihuahua: Estudio de Estado. Comisión Nacional para el Conocimiento y Uso de la Biodiversidad. México. Disponible en: https://www.biodiversidad.gob.mx/region/EEB/pdf/Chihuahua_Final_Web.pdf. (última consulta 10 de mayo de 2019). 
CONABIO (2018) Portal de geoinformación: Sistema Nacional de Información sobre Biodiversidad. Disponible en: http://www.conabio.gob.mx/informacion/gis/. (última consulta 24 de mayo de 2019).

Escobar, S. (2000) Diversidad de coleópteros coprófagos (Scarabaeidae: Scarabaeinae) en un mosaico de hábitats en la Reserva Natural Nukak, Guaviare, Colombia. Acta Zoológica Mexicana (nueva serie), $79,103-121$.

Fontúrbel, F. (2002) Rol de la coevolución planta-insecto en la evolución de las flores cíclicas en las angiospermas. Ciencia Abierta, 17, 11.

Furth, D. G., Jolivet, P. (2009) Flea beetle diversity of the Sierra Tarahumara, Copper Canyon, Mexico (Chrysomelidae: Alticinae). Research on Chrysomelidae, 2, 131-151. https://doi.org/10.1163/ej.9789004152045.1-299.46.

García, G. A. L., Morón, M. A., García, A. A., Arenas, L. D. O., Olivas, A. R., Carrasco, J. V. (2011) Especies fotófilas de Coleoptera Lamellicornia en la región de los Tascates, Sinaloa y Chihuahua (México). Boletín de la SEA, 49, 179-188.

Gómez-Beda, S. (2013) Importancia de los Scarabaeinae (Insecta: Coleoptera) como bioindicadores del estado de conservación en vegetación fragmentada de Tuxpan y Tamiahua, Veracruz, México. (Tesis de Maestría). Universidad Veracruzana, Facultad de Ciencias Biológicas y Agropecuarias. México.

Gómez, C., Gimenez, G., Munevar, A., Zurita, A. (2015) Estructura y composición de las comunidades de escarabajos estercoleros (Scarabaeidae: Scarabaeinae) en diferentes sistemas ganaderos del bosque atlántico de Argentina. Entomología Mexicana, 2, 591.

Gotelli, N. J., Chao, A. (2013) Measuring and estimating species richness, species diversity and biotic similarity from sampling data. 2nd ed. Waltham: Academic Press, $195 \mathrm{pp}$. https://doi.org/10.1016/b978-0-12-384719-5.00424-x

Guzmán-Mendoza, R., Calzontzi-Marín, J., Salas-Araiza, M. D., Martínez-Yáñez, R. (2016) La riqueza biológica de los insectos: análisis de su importancia multidimensional. Acta Zoológica Mexicana (nueva serie), 32, 37-379.

https://doi.org/10.21829/azm.2016.323971

Halffter, G., Rivera Cervantes, L. E., Halffter, V. (2015) Diversificación del grupo humectus del género Canthon (Coleoptera: Scarabaeidae: Scarabaeinae) en el occidente de México. Acta Zoológica Mmexicana (nueva serie), 31, 208-220. https://doi.org/10.21829/azm.2015.312542

Iglesias, L. E., Saumell, C. A., Fusé, L. A., Lifschitz, A. L., Rodriguez, E. M., Steffan, P. E., Fiel, C. A. (2005) Impacto ambiental de la Ivermectina eliminada por bovinos tratados en otoño, sobre la coprofauna y la degradación de la materia fecal en pasturas (Tandil, Argentina). RIA. Revista de Investigaciones Agropecuarias, 34, 83-103.

Instituto de Biología (IBUNAM) (2014) Colecciones Biológicas, Escarabajos. En Portal de Datos Abiertos UNAM Colecciones Universitarias (en línea), México, Universidad Nacional Autónoma de México. (última consulta 26 de junio de 2019).

Jiménez-Sánchez, E., Quezada-García, R., Padilla-Ramírez, J. (2013) Diversidad de escarabajos necrófilos (Coleoptera: Scarabaeidae, Silphidae, Staphylinidae y Trogidae) en una región semiárida del valle de Zapotitlán de las Salinas, Puebla, México. Revista de Biología Tropical, 61, 1475-1491. https://doi.org/10.15517/rbt.v61i3.11978

Jost, L. (2006) Entropy and diversity. Oikos, 113, 363-375. https://doi.org/10.1111/j.2006.0030-1299.14714.x

Martínez-Meyer, E., Sosa-Escalante, J. E., Álvarez, F. (2014) El estudio de la biodiversidad en México: ¿una ruta con dirección? Revista Mexicana de Biodiversidad, 85, 1-9. https://doi.org/10.7550/rmb.43248

Márquez-Luna, J. (2005) Técnicas de colecta y preservación de insectos. Boletín Sociedad Entomológica Aragonesa, 37, 385-408.

McCune, B., Grace, J. B. (2002) Analysis of ecological communities. Gleneden Beach, OR: MJM. 300 pp. 
McGavin, G. C. (2002) Insects, spiders and other terrestral arthropods. 2a ed. Editorial Dorling Kindersley. United States of America.

Mejía Narváez, M. D. C. (2018) Composición de las comunidades de coleópteros en sistemas silvopastoriles (acacia, aliso y pasto) y un bosque de referencia como indicadores de diversidad biológica en la Parroquia El Carmelo-Carchi-Ecuador (Tesis de Doctorado). Universidad Politécnica Estatal de Carchi, Facultad de Industrias Agropecuarias y Ciencias Ambientales. Ecuador.

Moreno, C. E., Barragán, F., Pineda, E., Pavón, N. P. (2011) Reanálisis de la diversidad alfa: alternativas para interpretar y comparar información sobre comunidades ecológicas. Revista Mexicana de Biodiversidad, 82, 1249-1261.

Núñez, I., González-Gaudiano, É., Barahona, A. (2003) La biodiversidad: historia y contexto de un concepto. Interciencia, 28, 387-393.

Pérez-De la Cruz, M., Hernández-May, M. A., la Cruz-Pérez, D., Sánchez-Soto, S. (2016) Scolytidae and Platypodidae (Coleoptera: Curculionidae) of two conservation areas in Tabasco, Mexico. Revista de Biología Tropical, 64 (1), 319-326.

https://doi.org/10.15517/rbt.v64i1.15931

Ribera, I., Foster, G. (1997) El uso de artrópodos como indicadores biológicos. Boletín de la Sociedad Entomológica Aragonesa, 20, 265-276.

Romero-Nápoles, J., Westcott, R. (2017) Familia Buprestidae, pp: 227-233) En: Cibrián-Tovar, D. (Ed.) Fundamentos de Entomología Forestal. Universidad Autónoma de Chapingo. Texcoco, Estado de México, México.

Sanabria, C., Armbrecht, I., Gutiérrez-Chacón, C. (2008) Diversidad de estafilínidos (Coleoptera: Staphylinidae) en cinco sistemas productivos de los Andes Colombianos. Revista Colombiana de Entomología, 34, 217-223.

Sánchez-Hernández, G., Gómez, B., Delgado, L., Rodríguez-López, M. E., Chamé-Vázquez, E. R. (2018) Diversidad de escarabajos copronecrófagos (Coleoptera: Scarabaeidae: Scarabaeinae) en la Reserva de la Biosfera Selva El Ocote, Chiapas, México. Caldasia, 40, 144-160. https://doi.org/10.15446/caldasia.v40n1.68602

Sánchez-Reyes, U. J., Niño-Maldonado, S., Meléndez-Jaramillo, E., Gómez-Moreno, V. D. C., BandaHernández, J. E. (2015) Riqueza de Chrysomelidae (Coleoptera) en el cerro el diente, San Carlos, Tamaulipas, México. Acta Zoológica Mexicana (nueva serie), 31, 10-22. https://doi.org/10.21829/azm.2015.311499

Sánchez-Soto, S. (2011 Conotrachelus dimidiatus Champion (Coleoptera: Curculionidae): el picudo de la guayaba (Psidium guajava L.) en Tabasco, México. Boletín del Museo de Entomología de la Universidad del Valle, 12, 17-18.

Thomas, C. D. (2013) Local diversity stays about the same, regional diversity increases, and global diversity declines. Proceedings of the National Academy of Sciences of the United States of America, 110, 19187-19188.

https://doi.org/10.1073/pnas.1319304110

Thorsten, F. K., Moon, A. R. (2019) Dung beetles. Current Biology, 551-567. https://doi.org/10.1016/j.cub.2019.05.027

Triplehorn, A. C., Johnson, F. N. (2005) Borror and Delongs Introduction to the Study of Insects. Thomson brooks/cole. United States of America, 263-267.

Trevilla-Rebollar, A., Deloya, C., Padilla-Ramírez, J. (2010) Coleópteros necrófilos (Scarabaeidae, Silphidae y Trogidae) de Malinalco, Estado de México, México. Neotropical Entomology, 39, 486495.

https://doi.org/10.1590/s1519-566x2010000400005

Trevizo, A. (2016) Caracterización del componente natural del rancho experimental Teseachi como insumo para su ordenamiento ecológico territorial. (Tesis de Maestría). Universidad Autónoma de Chihuahua, Facultad de Zootecnia y Ecología, Secretaría de Investigación y Posgrado. Chihuahua, México. 
UACH (2011) Rancho Tseachi, generador de investigación y santuario natural chihuahuense. Disponible en: http://portal.uach.mx/noticias/rectoria/2011/06/10/teseachi_investigacion/. (última consulta 20 de mayo de 2019).

Vellend, M. (2017) The biodiversity conservation paradox. American Scientist, 105, 94-101. https://doi.org/10.1511/2017.125.94

Zamora-Vuelvas, M. C., Ponce-Saavedra, J., Deloya-López, A. C. (2014) Scarabaeoidea (Insecta: Coleoptera) capturados con trampas en el cerro "El Aguila", Municipio de Morelia, Michoacán. Entomología Mexicana, 1, 519-524.

Zaragoza-Caballero, S. Z. (2015) Coleoptera: Cratomorphus halffteri, a new species of Mexico (Coleoptera: Lampyridae: Cratomorphini). Dugesiana, 18, 175-179.

Zhang, Z. Q. (2013) Phylum arthropoda. Zootaxa, 3703, 17-26.

Zhang, S. Q., Che, L. H., Li, Y., Liang, D., Pang, H., Ślipiński, A., Zhang, P. (2018) Evolutionary history of Coleoptera revealed by extensive sampling of genes and species. Nature Communications, 9 , 205.

https://doi.org/10.1038/s41467-017-02644-4 